\title{
Third-Harmonic Generation and Multi-Photon Excitation Fluorescence Imaging Microscopy Techniques for Online Art Conservation Diagnosis
}

\author{
EMILIO J. GUALDA, GEORGE FILIPPIDIS,* KRISTALIA MELESSANAKI, \\ and COSTAS FOTAKIS \\ Institute of Electronic Structure and Laser, Foundation of Research and Technology-Hellas, P.O. Box 1385, 71110, Heraklion, Greece (E.J.G., \\ G.F., K.M., C.F.); and Department Physics, University of Crete, Heraklion, Greece (C.F.)
}

\begin{abstract}
We present an appropriate methodology and results for using thirdharmonic generation (THG) modality for nondestructive high resolution imaging measurements of varnished structures in model painted artifacts. Detection takes place in the reflection mode, demonstrating the ability of the technique to be applied to the evaluation of original artworks. Furthermore, multi-photon excitation fluorescence images were obtained, providing complementary information related to the identification of the chemical composition of the artifacts.
\end{abstract}

Index Headings: Third-harmonic generation; THG; Multi-photon excitation fluorescence; MPEF; Imaging; Varnish; Artwork; Conservation.

\section{INTRODUCTION}

One major problem in the conservation of painted artifacts is the determination of the precise thickness of the outer layer of varnish. Natural varnish layers (resins) are historically one of the most usual materials employed to protect painted surfaces from atmospheric pollution and oxidation and to improve the appearance of the artifact. However, varnishes suffer from progressive deterioration due to continuous exposure to aggressive environmental conditions, giving rise to oxidation and polymerization products. ${ }^{1-3}$ This may affect the aesthetic appearance of the artifact and in some cases may diminish its lifetime. Therefore, varnishes usually need to be removed and replaced during regular conservation practice. Traditionally, this process occurs by applying chemical materials to dissolve the old varnish, an approach that is not easily controlled and may be harmful to the paintings.

Laser spectroscopic technologies have become an important tool in art conservation studies for determining the thickness of the varnish layers and for the on-line monitoring of the removal procedure. For thickness measurements, it is important to implement techniques that are nondestructive and highly sensitive in detecting small concentrations of pigments. Spectroscopic techniques such as laser-induced breakdown spectroscopy $(\mathrm{LIBS})^{2-4}$ or laser ablation and time-of-flight mass spectrometry (LAMS), ${ }^{5}$ which are based on a semidestructive approach, have been implemented. Furthermore, other techniques, such as laser-induced fluorescence (LIF) ${ }^{6}$ and optical coherence tomography (OCT) ${ }^{7-9}$ have been employed to measure the varnish thickness. More recently, THG spectroscopy has been introduced, providing promising results for the precise detection of transparent multilayer structures. ${ }^{10}$

Nonlinear imaging methodologies such as multi-photon excitation fluorescence (MPEF), second-harmonic generation (SHG), and third-harmonic generation (THG) are well-

Received 1 July 2008; accepted 11 December 2008.

* Author to whom correspondence should be sent. E-mail: filip@iesl forth.gr. established, nondestructive techniques that have been used as tools for the in vivo imaging and mapping of sub-cellular biological structures and processes ${ }^{11-14}$ and have also been employed for studies in the area of archaeology. ${ }^{15}$ Nonlinear imaging modalities present the capability of intrinsic threedimensionality, high axial resolution, the ability to section deep within the sample, and the reduction of "out-of-focal-plane" photobleaching in the specimens. These features are desirable for art conservation studies. Additionally, the use of femtosecond $(f s)$ lasers enables high peak powers for efficient nonlinear excitation, but at low enough energies so that samples are not damaged. Furthermore, in exploiting multiphoton processes, the wavelength of the illuminating source is located in the near-infrared (NIR) part of the spectrum. This helps further the reduction of risk for photodamage and makes filtering of the NIR excitation wavelengths from the fluorescent signal wavelengths extremely easy, improving the overall sensitivity of the detection system.

In previous work ${ }^{10}$ we have shown that the precise detection of different layers of natural and synthetic varnishes is feasible by employing an imaging system that combines three-photon excitation fluorescence (3PEF) and THG modalities in a single tool. THG is associated with the real part of $\chi^{(3)}$ susceptibility, and it is reinforced when the focus of the laser beam is at an interface between two media with different refractive indices. $^{12,16}$ This allows imaging based on THG to resolve otherwise transparent interfaces and inhomogeneities within the resolution of the confocal parameter and without the use of external dyes. Thus, THG has been used to image the interfaces between different layers of a transparent sample with a threedimensional (3D) microscopy capability, while the 3PEF signal was confined to the natural varnish layer. ${ }^{10}$ In our previous series of measurements, THG was measured in the forward direction, where the signal is stronger due to its coherent nature. This required the use of transparent samples. However, real painted artifacts are not transparent structures, including multi-layers of pigments, varnishes, and other materials, so for real applications the reflection mode of operation is imposed. In this study, we present the analysis of five different model painting samples showing the capability for the precise determination (resolution is on the order of a few micrometers) of the thicknesses of the protective varnish layers in painting materials using a combination of THG and MPEF imaging modalities in the reflection mode.

\section{EXPERIMENTAL}

The experimental setup is outlined in Fig. 1. The laser source used throughout this work was a diode-pumped Ytterbium- 


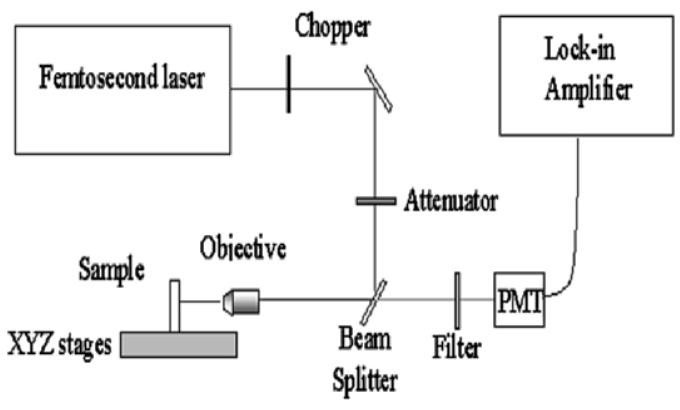

FIG. 1. Schematic representation of the experimental setup.

doped solid state laser (t-Pulse, Amplitude Systemes), with a repetition rate of $50 \mathrm{MHz}$, operating at a central wavelength of $1028 \mathrm{~nm}$. The average power at the laser output was $1 \mathrm{~W}$ and the pulse duration was $200 \mathrm{fs}$. The average power at the sample plane was less than $20 \mathrm{~mW}$ in order to avoid ablation effects and burnings into the specimens. A dichroic mirror was used to send the light to the sample. A two-lens arrangement served to expand the laser beam to fill the aperture of the objective lens (Carl Zeiss, Plan-Apochromat 100×, N.A. 1.4).

The same setup was used to measure both THG and MPEF signals, in distinct sets of measurements. A $340 \mathrm{~nm}$ colored glass filter (Hoya U340) and a short pass filter at $650 \mathrm{~nm}$ (SPF $650 \mathrm{~nm}$ CVI) were used for the THG and MPEF measurements, respectively. The reflected signal was measured with a photomultiplier tube (PMT Hamamatsu H9305-04) connected to a lock-in amplifier (SR810 Stanford Research Systems). The sample position was controlled by an $x-y-z$ motorized stage (Standa 8MT167-100) with minimum steps of $1 \mu \mathrm{m}$ in each direction.

Sample Preparation. Pigments, the main component of painted artworks, are derived from a wide variety of organic and inorganic substances, both natural and artificial. ${ }^{1}$ They are granular solids and a small amount of them is sufficient to give color to the paint. Oil, acrylic, and egg are some of the main binding materials (mediums) that hold together pigment particles in paints and fix them to the painting surface. The final touch to an artwork is given with one or more layers of varnishes. Varnish is a solution of a resin in a solvent, which is used to protect and preserve the artifact from atmospheric pollution; it also improves the aesthetic appearance by providing an even surface finish, brilliance, and depth to the colors.

On round glass plates $35 \mathrm{~mm}$ in diameter and $\sim 45 \mu \mathrm{m}$ thick (Marienfeld), five different model samples reproducing the properties of a real artwork have been prepared. These samples represent the three main categories of painted artworks, oil paint from the Renaissance period, egg tempera from the Byzantine era, and acrylic paint from modern time.

The first two samples were prepared with ready-made acrylic paint (Rowney CRYLA, artistic acrylic color). Sample \#1 is lemon yellow, a barium chromate $\left(\mathrm{BaCrO}_{4}\right)$, and sample \#2 is an ochre yellow $\left(\mathrm{Fe}_{2} \mathrm{O}_{3} * \mathrm{nH}_{2} \mathrm{O}, \mathrm{SiO}_{2}, \mathrm{Al}_{2} \mathrm{O}_{3}\right)$. Sample \#3 is an oil color paint made by mixing ochre yellow $\left(\mathrm{Fe}_{2} \mathrm{O}_{3} * \mathrm{nH}_{2} \mathrm{O}\right.$, $\mathrm{SiO}_{2}, \mathrm{Al}_{2} \mathrm{O}_{3}$ ) pigment with linseed oil. In samples \#4 and \#5 egg yolk was employed as a binding medium. Sample \#4 contains titanium white $\left(\mathrm{TiO}_{2}\right)$ pigments and sample \#5 contains barium chromate $\left(\mathrm{BaCrO}_{4}\right)$ pigments. All the above model painting samples were covered with one layer of natural (a)
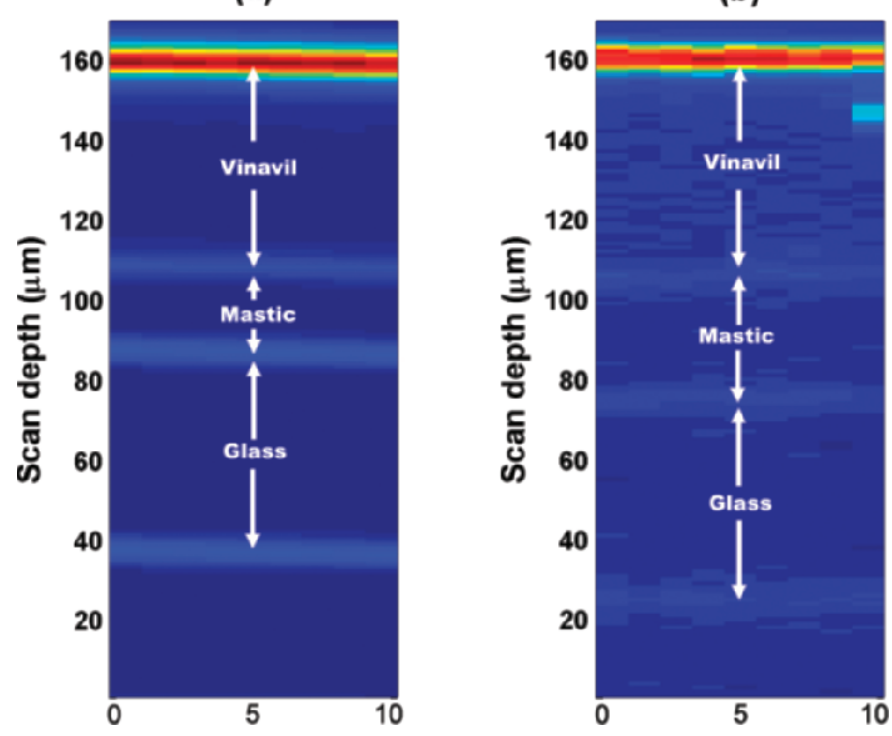

FIG. 2. Sectioning of a transparent sample containing a layer of synthetic varnish and a layer of natural varnish: (a) THG in transmission mode and (b) THG in reflection mode. The lateral dimension of the scanning area of the recorded images is $10 \mu \mathrm{m}$ with $1 \mu \mathrm{m}$ resolution.

varnish (colophony dissolved in white spirit), in order to produce multilayer structures.

Finally, for testing reasons a transparent sample (\#6) was studied, containing one layer of natural varnish (mastic dissolved in turpentine) and another layer of synthetic varnish (vinavil, a polyvinyl acetate homopolymer, dissolved in water).

All the samples were cast to produce layers of even thickness. The measurements have been performed on fresh samples.

Backward-Reflected Third-Harmonic Generation Signal. In order to evaluate the possibility for the precise discrimination of the different layers of material (of a real artifact) through the detection of THG modality in the reflection mode, a transparent sample (sample \#6) has been analyzed and compared in both transmission and reflection modes. Figure $2 \mathrm{a}$ depicts a THG image detected in the forward direction using the setup described in Ref. 10 but scanning the sample with an $x-y-z$ motorized stage. Figure $2 b$ shows THG signals obtained in the reflection mode setup from the same sample. (a)

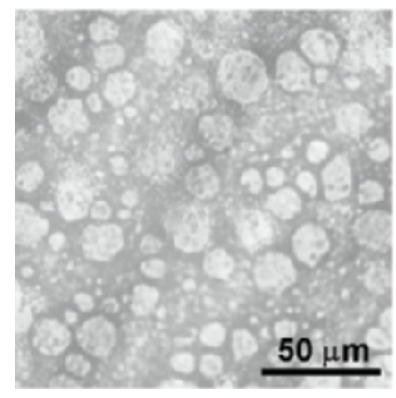

(b)

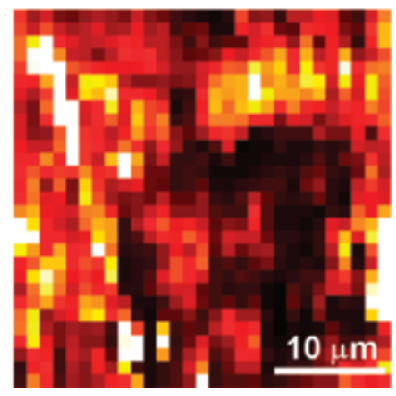

FIG. 3. (a) White light picture of sample that contains barium chromate in an acrylic medium. (b) Smaller area of the same sample imaged through THG in reflection mode with $1 \mu \mathrm{m}$ resolution. White color corresponds to the maximum and black color to the minimum THG intensity signals. 
(a)

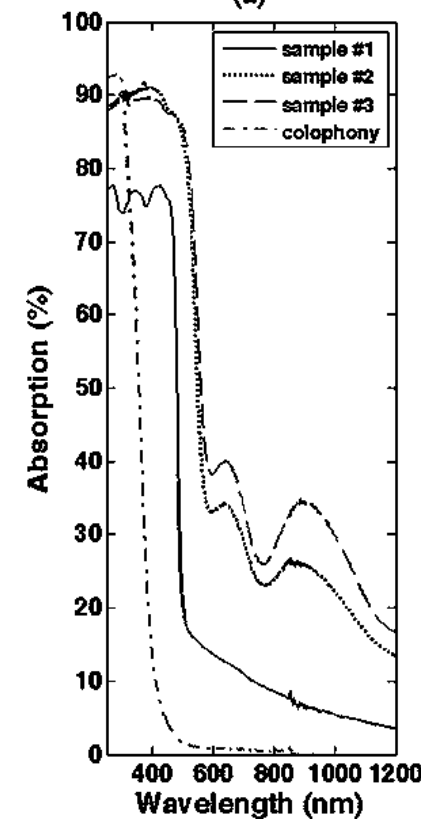

(b)

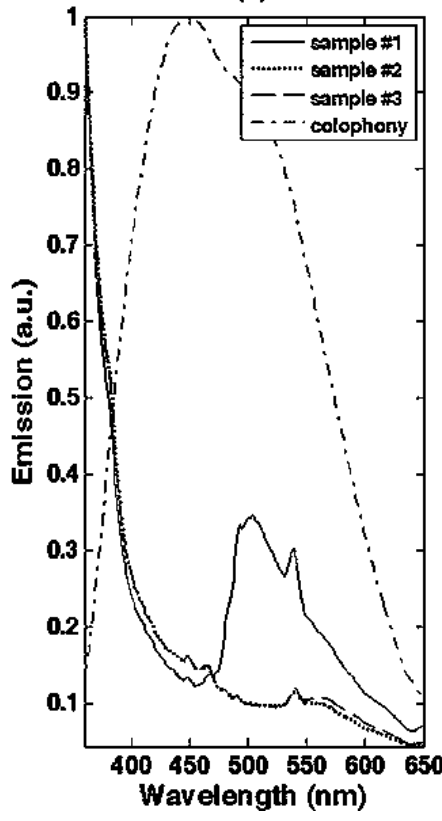

(c)

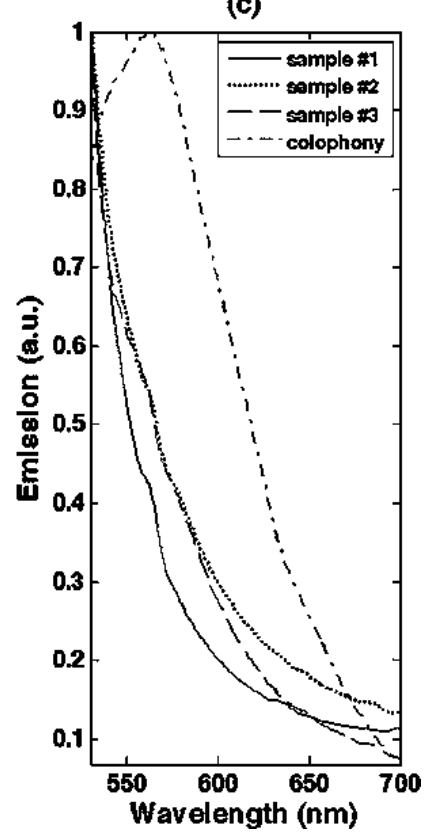

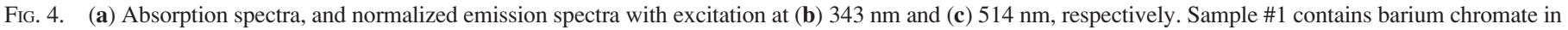

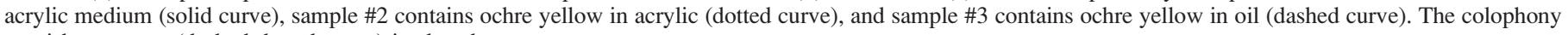
varnish spectrum (dashed-dotted curve) is also shown.

Although the THG signal is lower in reflection mode (Fig. 2b) compared with transmission mode (Fig. 2a), four different layers can be distinguished, indicating the interfaces between the different materials. The upper one corresponds to the airsynthetic varnish interface and the lower one corresponds to the glass-air interface. The other two represent the synthetic varnish-mastic and mastic-glass interfaces, respectively. The thicknesses of the mastic and synthetic varnish layers measured in reflection mode compared with those measured in transmission mode are: $22 \mu \mathrm{m}$ in transmission and $29 \mu \mathrm{m}$ in reflection mode for the mastic layer, and $\sim 54 \mu \mathrm{m}$ for the synthetic varnish layer in both modes. The measured thickness of the glass is always around $45 \mu \mathrm{m}$. It should be mentioned that the THG signal on each interface presents a Gaussian-like shape and the estimated thickness is the distance between the maximal values of each interface. (a)

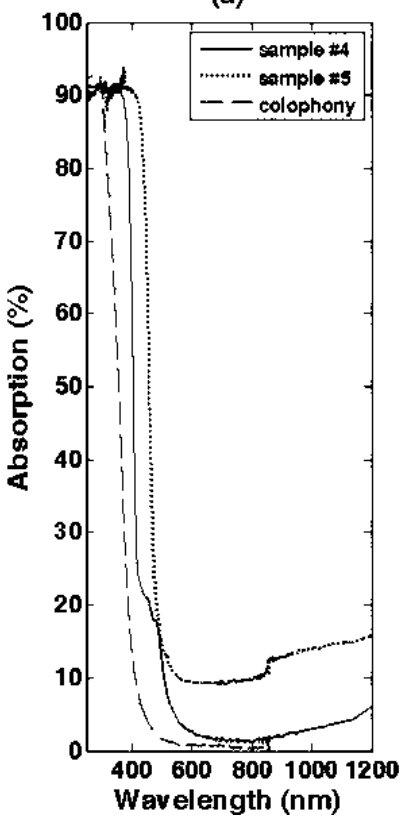

(b)

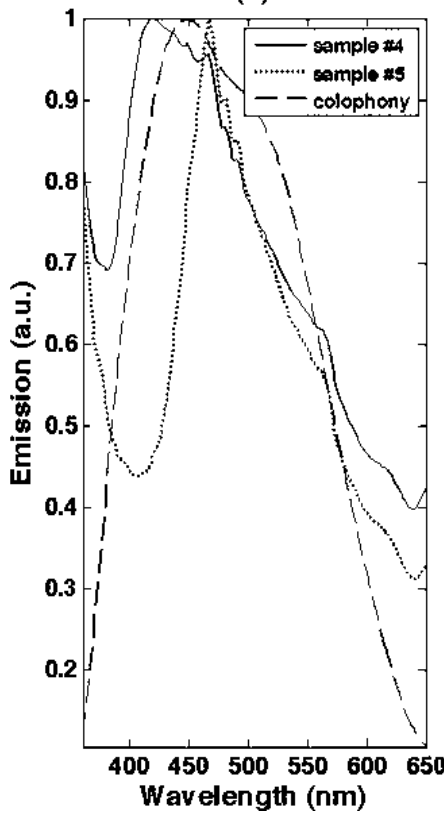

(c)

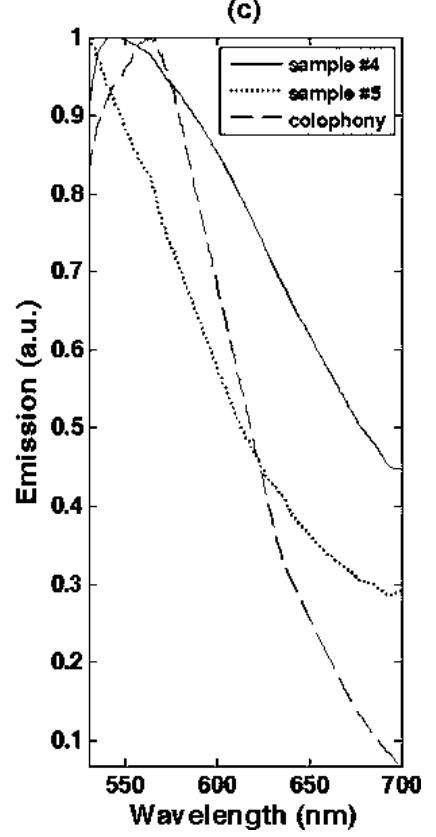

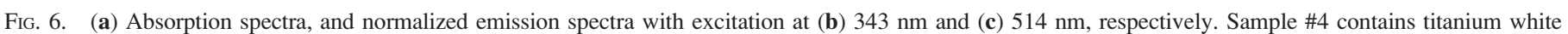

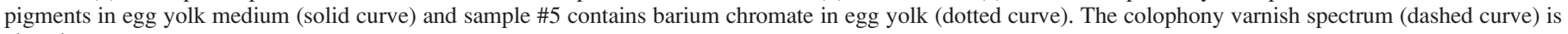
also shown. 
(a)

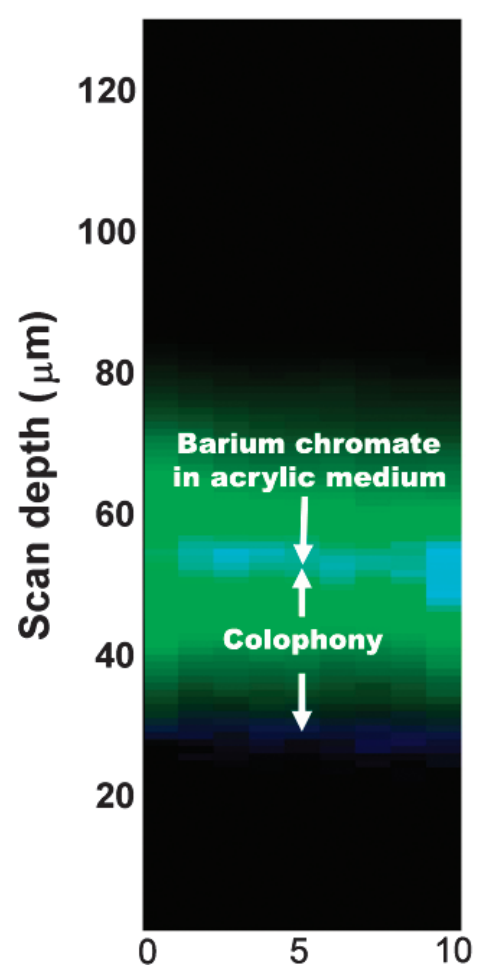

(b)

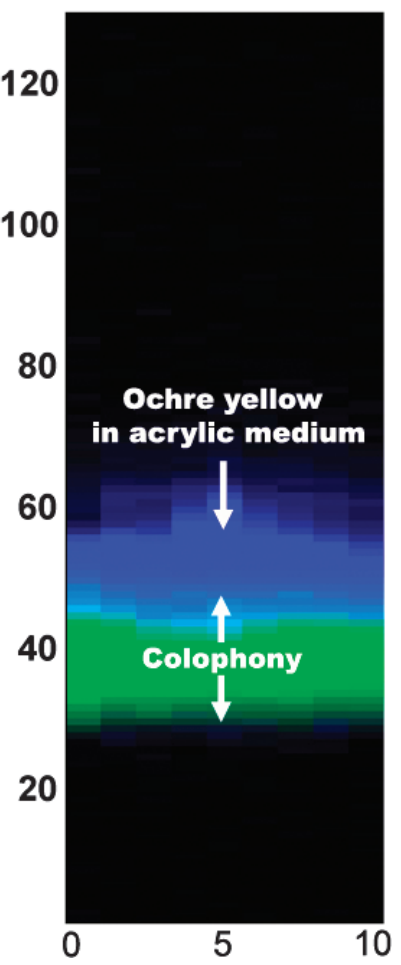

(c)

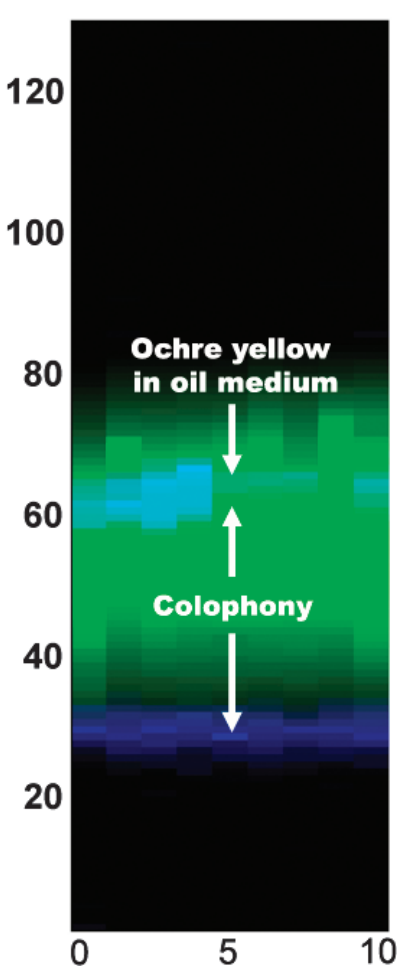

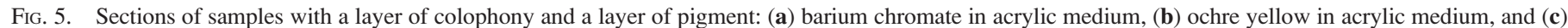

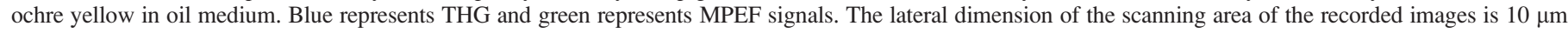
with $1 \mu \mathrm{m}$ resolution.

Due to technical reasons, the THG data in reflection mode and in transmission mode were obtained in distinct sets of measurements at different time periods. Moreover, slight differentiations of the thicknesses of the varnish layers is expected owing to the casting preparation method of the sample. Therefore, the small deviation of the measured thickness of the mastic layer can be attributed to the fact that the scanning procedure was performed at different positions on this kind of sample.

It is important to notice that this sample (\#6) is made by using uniform materials, without the use of any pigment, so the interface between media can be detected by a uniform THG signal. Also, both synthetic varnish and natural resins (mastic or colophony) are completely transparent in the infrared range and in a wide part of the visible range of wavelengths.

In the following subsections we analyze the nonlinear behavior, in terms of THG signal and multiphoton fluorescence, of five different model samples. These simulations of real artworks are composed of different kinds of binding mediums and pigments, covered with the same natural varnish protective material (colophony).

Oil and Acrylic Binding Mediums. In Fig. 3a is shown a picture of a sample containing barium chromate particles in an acrylic medium. It can be observed that the size of these particles ranges from submicrometer to several micrometers.

One consequence of the addition of pigments is that the interfaces between different layers are no longer homogeneous. A scan of the surface of the sample shows, in Fig. 3b, how the intensity of the THG signal is position dependent. Due to the coherent nature of THG, most of the signal is created in the forward direction, but the presence of metallic components and nanoparticles also makes possible the detection of a THG signal in the reflection mode. ${ }^{17}$

In order to study the effects of various pigments and binders, three different model samples have been analyzed using nonlinear imaging techniques. Two different kinds of binders (a)

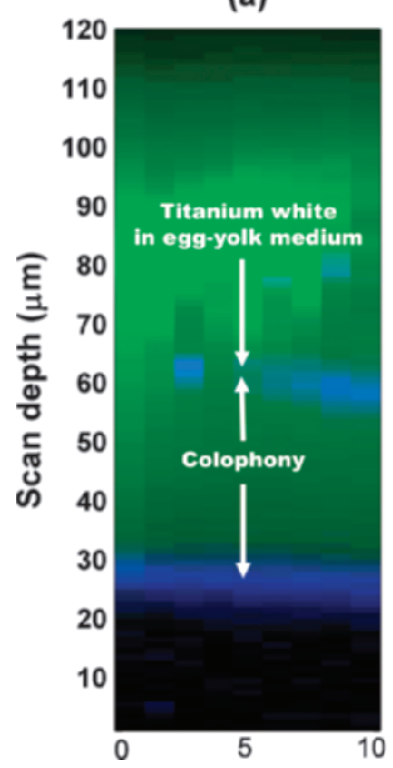

(b)

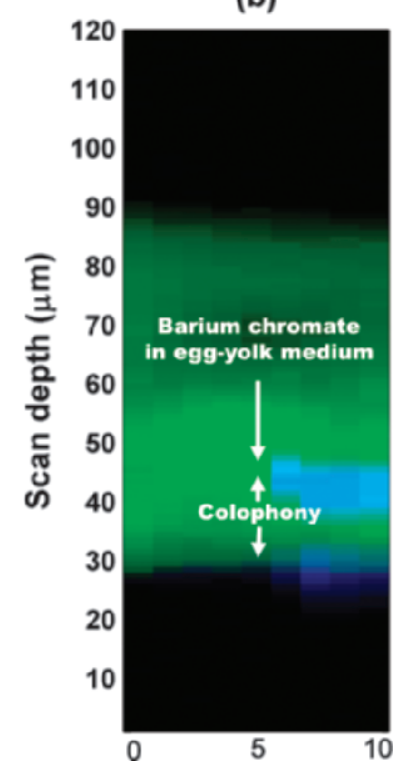

FIG. 7. Sections of samples with a layer of colophony and a layer of pigment in egg yolk medium: (a) titanium white and (b) barium chromate. Blue represents THG and green represents MPEF signals. The lateral dimension of the scanning area of the recorded images is $10 \mu \mathrm{m}$ with $1 \mu \mathrm{m}$ resolution. 
are taken into account, acrylic and oil, mixed with barium chromate and ochre yellow pigments. Sample \#1 is barium chromate and sample \#2 is ochre yellow in acrylic medium. Sample \#3 is ochre yellow with linseed oil.

In Fig. 4 the absorption and emission spectra for these three samples are presented, along with the data corresponding to colophony. The absorption spectra (Fig. 4a) were measured with a UV/Vis Spectrometer (Perkin Elmer-Lambda 950). The emission spectra (Figs. $4 \mathrm{~b}$ and $4 \mathrm{c}$ ) were taken using a fluorometer (Jobin Yvon Horiba FluoroMax- P) with excitation wavelengths at $343 \mathrm{~nm}$ and $514 \mathrm{~nm}$, respectively. Figures $4 \mathrm{~b}$ and $4 \mathrm{c}$ present the normalized measured fluorescence intensities of the samples. The fluorescence intensity of colophony is comparable with the intensities of the other three materials at $343 \mathrm{~nm}$ (Fig. 4b), but it is much weaker at $514 \mathrm{~nm}$ (Fig. 4c).

It can be observed that all the materials present high absorption coefficients at UV wavelengths, while the absorption in the visible range (at $514 \mathrm{~nm}$ ) depends strongly on the pigment used. At $514 \mathrm{~nm}$ ochre yellow presents $80 \%$ absorption, independently of the binding medium used, while barium chromate only reaches $17 \%$. Moreover, these materials present absorption at the fundamental laser wavelength of 1028 nm (5\% for sample \#1 and around 25\% for samples \#2 and \#3). Thus, while for colophony (natural varnish) mainly threephoton absorption is important, for the other painting materials the absorption process is a combination of one-, two-, and three-photon absorption. Consequently, it is easy to damage the painting samples by burning. From our experiments it has been observed that the damage threshold is not reached using average powers at the sample plane of less than $20 \mathrm{~mW}$ (less than $0.4 \mathrm{~nJ}$ per pulse). We have to note that nonlinear signals appear in a small incident intensity window, below which the signal is too low and above which the sample is damaged. Therefore, the restricted numbers of data points within this window are not enough to prove unambiguously the nonlinear dependence of fluorescence on the input laser intensity. However, the minimal absorption of colophony in the visible and infrared spectral regions indicates that the main contribution to the fluorescence process is due to three-photon excitation.

Figure 5, shows the two-dimensional section of the three samples described above, with a layer of colophony of different thicknesses on top of each. Both the THG (blue) and MPEF (green) signals are depicted. The vertical axis corresponds to the scanning depth with around $3 \mu \mathrm{m}$ resolution and the horizontal axis corresponds to ten different positions $1 \mu \mathrm{m}$ apart.

From the reflected THG signal the air-colophony and the colophony-pigment interfaces can be clearly distinguished for the three samples. The measured thicknesses of the varnish (colophony) layers are 24, 21, and $35 \mu \mathrm{m}$ for samples \#1, \#2, and \#3, respectively. As has been already pointed out (Fig. 3b), there is a difference between the THG signal obtained from the two interfaces: while the former (air-colophony) is a continuous line since colophony varnish is a uniform material, the latter (colophony-pigment) is not homogeneous and is only created at the pigment particles. The refractive index mismatch is higher between ochre yellow and the colophony at their interface than the one between barium chromate and colophony. ${ }^{1}$ Consequently, keeping the same illuminating conditions, the efficiency of the THG process is higher for the ochre yellow-colophony interface (sample \#2) than that for the barium chromate-colophony interface (sample \#1). That is the reason why the second interface is more clearly distinguished in Fig. 5b than in Fig. 5a. Additionally, it is worthwhile to notice that, due to the strong absorption of the pigments for the three samples, only the detection of the outer structures of the model paintings is feasible. The two first interfaces (air-varnish, varnish-painting) via THG imaging measurements and a portion of the pigmented layer through multi-photon fluorescence are detectable. This fact makes impossible the use of the transmission configuration, even for testing purposes.

Multi-photon excitation fluorescence signals present almost the same level of intensity for the varnish and for the pigmented material for sample \#1 (Fig. 5a). From this sample it can be pointed out that MPEF measurements alone are not enough for measuring the thickness of varnish. The detected THG signals provide the proper information for the precise thickness determination of the varnish protective layer.

Moreover, comparing the MPEF signals from samples \#2 and \#3 (both containing ochre yellow pigments), it can be observed that the main contributor to the fluorescence is the binding media. While oil creates a higher level of signal (Fig. 5c), acrylic is not generating any (Fig. 5b). The recorded MPEF signal from sample \#2 (acrylic binding medium) arises from the colophony varnish. This observation is in agreement with previously reported studies showing that the fluorescence detected in paint based on metallic compounds is generated by the binding mediums, while the pigment actually may inhibit the generation of fluorescence. ${ }^{18}$

Egg Yolk Binding Medium. Another type of artistic paint is tempera. In this technique the binding medium in which the pigment is embedded is egg yolk.

In order to analyze the behavior of paintings containing egg yolk, two different samples have been prepared. Sample \#4 contains titanium white $\left(\mathrm{TiO}_{2}\right)$ pigments and sample \#5 contains barium chromate $\left(\mathrm{BaCrO}_{4}\right)$ pigments. Figure 6a shows the absorption spectra and Figs. $6 \mathrm{~b}$ and $6 \mathrm{c}$ show the emission spectra exciting at $343 \mathrm{~nm}$ and $514 \mathrm{~nm}$, respectively. It can be observed that the absorption of the materials is higher at $343 \mathrm{~nm}(\sim 91 \%)$ than at $514 \mathrm{~nm}(\sim 10 \%)$. The fluorescence curves (Figs. $6 \mathrm{~b}$ and $6 \mathrm{c}$ ) show that the contribution to the MPEF is due to both two- and three-photon absorption.

Figure 7 shows the combination of THG (blue) and MPEF (green) measurements for these two samples. On top of the pigments a layer of colophony varnish has been introduced. As in the previous measurements, the lateral dimension of the scan is $10 \mu \mathrm{m}$. From the THG measurements it can be observed that the thickness of the varnish layer is $35 \mu \mathrm{m}$ for sample \#4 containing titanium white (Fig. 7a). For sample \#5, with barium chromate (Fig. $7 \mathrm{~b}$ ), the thickness is $12 \mu \mathrm{m}$. In the case of sample \#4 some inhomogeneities inside the painting material also generate THG signal. However, the main difference from the samples analyzed in the previous section containing oil or acrylic as a binding media is the behavior of the MPEF signal. The level of the signal arising from the painting layer is much stronger than the signal from the colophony. Thus, while the THG signal gives the information about the position of the layer interfaces, MPEF would provide complementary information about the painting technique that has been used, since, compared with the fluorescence of the colophony, the total fluorescence coming from the painting 
material is higher. Consequently, this technique represents a new qualitative way to detect the thicknesses of varnish protective layers. Furthermore, it allows the discrimination, rather than the determination, of the composition between different painting media.

\section{CONCLUSION}

The possibility of measuring THG signals in the reflection mode in model painted materials has been demonstrated. The precise determination of the thickness of a varnish protective layer through THG imaging measurements was achieved. Five different widespread painted materials were studied. THG imaging modality is an appropriate technique for realistic diagnostic applications in painted artifacts, since it provides minimum sample disturbance. By combining the information recorded through THG with that obtained from MPEF measurements, complementary information such as discriminating the chemical composition of painting materials and understanding the painting techniques employed can be extracted using the same simple setup.

Additionally, since the ablation and removal of varnish surfaces is possible using femtosecond lasers, ${ }^{19}$ we anticipate that these diagnostic nonlinear imaging modalities, which present comparable accuracy with other techniques (e.g., OCT), can be integrated in a single novel instrument with the capabilities of diagnosis and laser cleaning for art restoration purposes. The compact size and the reduced dimensions of the employed excitation source allows its integration in a transportable device with the capabilities of in situ laser diagnosis and cleaning of painted artworks. The construction of a laser cleaning apparatus that integrates these nondestructive diagnostic modalities has the potential to provide precise online control of the cleaning procedure of artifacts and can help to avoid damage to the exposed painted surface.

\section{ACKNOWLEDGMENTS}

This work was supported by the UV Laser Facility operating at IESLFORTH under the European Commission "Improving Human Research Potential" program (RII3-CT-2003-506350) and by the Marie Curie Transfer of Knowledge project "NOLIMBA" (MTKD-CT-2005-029194).

1. R. J. Gettens and G. L. Stout, Painting Materials (Dover, New York, 1966).

2. K. Melessanaki, C. Stringari, C. Fotakis, and D. Anglos, Laser Chem. 2006, 42709 (2006).

3. C. Fotakis, D. Anglos, V. Zafiropulos, S. Georgiou, and V. Tornari, Lasers in the Preservation of Cultural Heritage: Principles and Applications (Taylor \& Francis, London, 2006).

4. D. Anglos, Appl. Spectrosc. 55, 186 (2001).

5. R. Torres, M. Jadraque, M. Castillejo, and M. Martín, Lasers in the Conservation of Artworks (LACONA) V Proceedings, Springer Proceedings in Physics 100, 285 (2005).

6. A. Nevin, S. Cather, D. Anglos, and C. Fotakis, Anal. Chim. Acta 573, 341 (2006).

7. P. Targowski, M. Gora, and M. Wojtkowski, Laser Chem. 2006, 10647 (2006).

8. T. Arecchi, M. Bellini, C. Corsi, R. Fontana, M. Materazzi, L. Pezzati, and A. Tortora, Opt. Spectrosc. 101, 23 (2006).

9. P. Targowski, B. Rouba, M. Góra, L. Tymińska-Widmer, J. Marczak, and A. Kowalczyk, Appl. Phys. A 92, 1 (2008).

10. G. Filippidis, E. J. Gualda, K. Melessanaki, and C. Fotakis, Opt. Lett. 33, 240 (2008).

11. W. Denk, J. H. Strickler, and W. W. Webb, Science (Washington, D.C.) 248, 73 (1990).

12. J. Squier, M. Muller, G. Brakenhoff, and K. R. Wilson, Opt. Exp. 3, 315 (1998).

13. S. W. Chu, S. Y. Chen, T. H. Tsai, T. M. Liu, C. Y. Lin, H. J. Tsai, and C. K. Sun, Opt. Exp. 11, 3093 (2003).

14. D. Débarre, W. Supatto, and E. Beaurepaire, Opt. Lett. 30, 2134 (2005).

15. I. G. Cormack, P. Losa-Alvarez, L. Sarrado, S. Tomas, I. Amat-Roldan, L. Torner, D. Artigas, J. Guitart, J. Pera, and J. Ros, J. Archaeol. Sci. 34, 1594 (2007).

16. T. Y. F. Tsang, Phys. Rev. A 52, 4116 (1995).

17. D. Carroll and X. H. Zheng, Eur. Phys. J. D 5, 135 (1999).

18. T. Miyoshi, Jpn. J. Appl. Phys. 27, 627 (1988).

19. P. Pouli, I. A. Paun, G. Bounos, S. Georgiou, and C. Fotakis, Appl. Surf. Sci. 254, 6875 (2008). 